\title{
Physicienne, bien dans sa peau, bien dans son métier
}

Sandrine Morin (sandrine.morin@univ-rouen.fr)

Membre de la Commission « Femmes et Physique » de la SFP

Laboratoire de Biophysique et Biomatériaux, MERCl, EA 3829, Université de Rouen, Centre universitaire d’Évreux, BP 281, 27002 Évreux Cedex

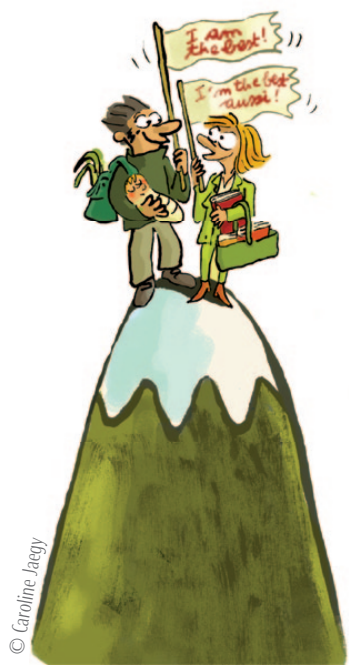

À Marseille, lors du Congrès général de la SFP, le minicolloque de la Commission Femmes et Physique (F\&P) ${ }^{(1)}$ a réuni le 4 juillet 2013 une soixantaine de participants, dont une dizaine d'hommes. Au programme : une conférence sur les carrières des physiciennes, une table ronde et la présentation du bilan de I'enquête INTEGER menée au sein du CNRS.

(1) www.sfpnet.fr/index.php?page $=$ tpage $\&$ id $=21$

(2) Projet ANR TRAJUNI,

www.cso.edu/page_pole.asp?ppol_id=169\&pol_id=20

(3) F. Pigeyre et M. Sabatier, « Les carrières des femmes à l'université : une synthèse de résultats de recherche dans trois disciplines ", http://pmp.revues.org/4197

(4) LURE = Laboratoire pour l'Utilisation du

Rayonnement Électromagnétique (Orsay)

(5) CINaM = Centre Interdisciplinaire de

Nanoscience de Marseille

(6) INstitutional Transformation for Effecting Gender Equality in Research

(7) www.cnrs.fr/mission-femmes/INTEGER

\section{Les carrières des physiciennes à l'Université : quelques motifs d'inquiétude}

Frédérique Pigeyre, professeure en Sciences de gestion à l'Université Paris-Est Créteil, a présenté les résultats d'une étude réalisée grâce aux bases de données du ministère et de nombreux entretiens ${ }^{(2)}$. Ce travail a permis une analyse quantitative (mesure des écarts hommes/femmes) et qualitative (contrainte familiale, organisationnelle, vision du métier...) des carrières universitaires dans plusieurs disciplines, ainsi qu'une observation des évolutions de 1976 à $2006^{(3)}$.

Comme dans tous les secteurs d'activités et dans tous les pays, la féminisation augmente chez les enseignants-chercheurs (passage de 17 à $22 \%$ de la proportion de femmes sur 20 ans). En physique, la situation est fragile, la proportion de femmes a diminué sur la dernière année.

Malgré cette féminisation, le passage des femmes au grade de professeure est une des difficultés récurrentes dans toutes les disciplines (en physique, en 2009-2010, $27 \%$ des maîtres de conférences mais seulement $12 \%$ des professeurs étaient des femmes). C'est ce qu'on appelle le « plafond de verre ", cette barrière invisible rencontrée par les femmes. Frédérique Pigeyre a présenté plusieurs pistes d'explication :

- Historiquement, les femmes ont eu un accès tardif à l'université (19 siècle) ; elles ont été longtemps pas ou peu éduquées.

- Les femmes font des choix différents dans leur carrière. Si on regarde la « division " du travail à l'université, on observe que les femmes participent plus aux tâches pédagogiques ou administratives et se retrouvent, par conséquent, moins investies dans la recherche.
- L'arbitrage entre la vie personnelle et professionnelle joue également un rôle. La charge de la famille pèse, toujours, beaucoup plus sur les épaules des femmes. - L'effet de la discrimination indirecte (réunions le soir à 19 heures...)

- L'autocensure persistante chez les femmes.

Pour la carrière universitaire, les critères mis en avant sont la productivité scientifique et le prestige. C'est une représentation très " masculine " de ce qui fait un bon scientifique. "Un bon scientifique doit être déchargé des contingences matérielles et se consacrer uniquement à sa recherche. "Ces considérations mènent à une évaluation "genrée " des carrières.

En physique, l'âge de recrutement moyen (32 ans) est le même pour les hommes et les femmes. Il y a quelques années, le nombre de publications chez les femmes recrutées était inférieur à celui des hommes, ce n'est plus le cas aujourd'hui. Le recrutement devient donc plus difficile pour les femmes ! Frédérique Pigeyre a montré l'importance du fonctionnement des commissions de spécialistes sur ce recrutement (prise en compte ou non des maternités dans le bilan des publications, utilisation de critères sexués comme " avoir du mordant »...). Dans ce sens, les enquêtes menées auprès des membres des commissions montrent qu'il est important de maintenir la mixité des jurys.

Pour le passage au grade de professeur, il y a pénurie de candidatures en général et particulièrement de candidatures féminines. En effet, ce poste est souvent considéré comme trop contraignant par les femmes elles-mêmes. Elles sont aussi moins encouragées par leur équipe et moins intégrées dans des réseaux. En physique, un homme est promu professeur au bout de douze ans 


\section{Femmes et science}

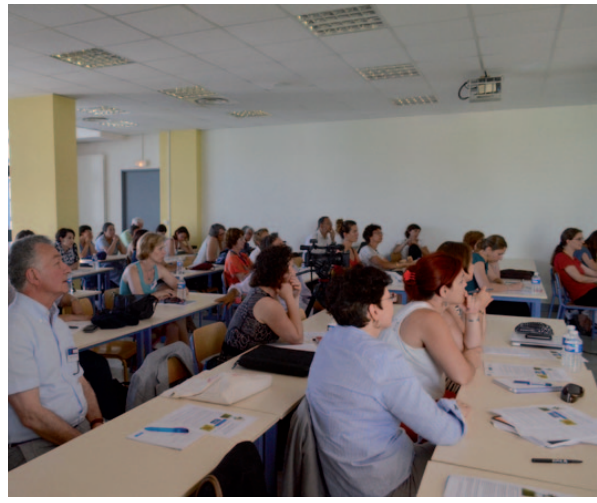

L'assistance

de carrière en moyenne. La chance de passer professeure est beaucoup plus faible pour les femmes, quelle que soit leur ancienneté.

En physique, Frédérique Pigeyre a pu observer une amélioration du recrutement des femmes, mais une évolution des carrières beaucoup plus lente que dans d'autres disciplines : le plafond de verre est tenace !

\section{Table ronde : « Physicienne, bien dans sa peau et bien dans son métier »}

La commission F\&P a réuni des physiciennes qui ont réussi dans différents métiers après une thèse et, souvent, plusieurs postdoctorats. Les invitées ont présenté leur parcours, leur évolution de carrière, avant de participer à un débat.

Marielle Fontaine, responsable " méthodes " dans l'aéronautique chez Bonnans à Marseille, a toujours été motivée par l'industrie. Elle a souligné l'importance d'appartenir à un réseau et "d'avoir et de conserver une volonté de fer!".

Dominique Chandesris, directrice de recherche (DR) au CNRS, directrice du Laboratoire de Physique des Solides à Orsay, ex-directrice scientifique adjointe du département Mathématiques, Physique et Univers du CNRS. Elle est entrée jeune au CNRS (en 1977), ce qui n'est plus le cas pour les femmes aujourd'hui, au LURE ${ }^{(4)}$. "Ce laboratoire avait déjà une forte proportion de femmes, donc les jeunes doctorantes avaient envie d'y aller! Cet environnement a constitué un cercle vicieux positif. " Elle est consciente des discriminations indirectes qui existent, même si elle n'en a jamais souffert.

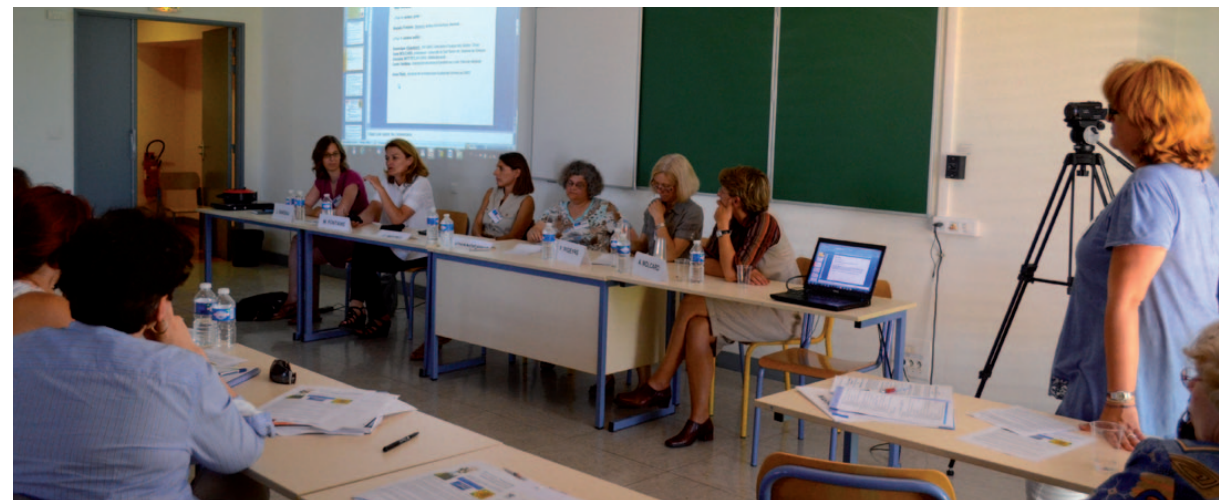

Les invitées. De gauche à droite, le long de la table : L. Sandeau, M. Fontaine, C. Mottet, D. Chandesris, F. Pigeyre et A. Molcard. À droite de la photo, S. Morin. (Photos : F. Vedel).

Anne Molcard, professeure en océanographie physique à l'Université de Toulon. Après l'obtention d'un poste, huit ans après sa thèse, elle a vite passé son habilitation à diriger les recherches (HDR) pour postuler comme professeure et être mieux reconnue au sein de l'université. "La place des femmes est plus difficile dans de nombreux secteurs d'activité, alors autant exercer un métier passionnant!"

Christine Mottet, DR CNRS au $\mathrm{CINaM}^{(5)}$ à Marseille. Après un post-doctorat en Italie, elle entre au CNRS à Marseille, puis passe DR neuf ans plus tard. "Au CNRS, on n'est pas obligé de passer par une mobilité lors d'une promotion, ce qui est plus simple pour la vie familiale."

Laure Sandeau, titulaire d'une thèse en biophysique, aujourd'hui enseignante en classe préparatoire au Lycée Thiers de Marseille. Après sa thèse, elle a choisi un poste en lycée (6 ans à Paris), puis a travaillé dans une start-up à Marseille pour se rapprocher de son mari. Elle retourne en lycée après son deuxième enfant. "Mes choix de carrière ont suivi les clichés féminins et se sont faits en fonction de ma vie de famille. "

Au cours du débat, il y a eu de nombreuses réactions sur la "contrainte " que représente la candidature sur un poste de professeure : "Postuler à un poste de professeure demande souvent de déménager. C'est un problème pour bouger si on est en couple, car il faut trouver deux postes!"; "Il faut changer les mentalités, car aujourd'hui c'est plus souvent la femme qui suit son mari que l'inverse!"

L'auditoire de physiciens et physiciennes expérimentés a confirmé l'importance des réseaux, que ce soit à l'université ou en entreprise : "Ce n'est souvent pas un comportement naturel pour les femmes de se mettre en avant. "; "Dans les propositions pour les postes, les noms de femmes ne viennent pas naturellement!"

Des témoignages ont également confirmé l'autocensure des femmes lorsqu'elles sont sollicitées pour des postes à responsabilités : "C'est difficile de trouver une candidate car elles disent: "Cela va dépasser mon niveau de compétences.” "

L'auditoire a pointé du doigt le nombre de postdoctorats demandés pour un poste (4 à 5 parfois !), ce qui représente une discrimination indirecte : "L'âge moyen de recrutement à 32 ans est très défavorable aux filles. Il faut recruter plus jeune (homme et femme). Quel homme suit sa femme à l'étranger pour un postdoctorat? Il faut que la communauté de femmes scientifiques essaie d'influer sur l'évolution de ce critère de recrutement. "

Pour conclure, on retiendra cette intervention : "La carrière se construit dès le début. La façon dont la femme est perçue est très importante! Dans les équipes, il y a un gap entre la façon dont on perçoit les hommes et les femmes."

\section{Résultats de l'enquête INTEGER ${ }^{(6)}$}

Anne Pépin, directrice de la mission pour la place des femmes au CNRS, a présenté le bilan de l'enquête INTEGER menée au sein du CNRS depuis 2011 ${ }^{(7)}$. Ces résultats feront l'objet d'un prochain article dans Reflets de la physique.

$\mathrm{La}$ transcription de l'intégralité des échanges peut être consulté sur le site de la Commission $\mathrm{F} \& \mathrm{P}^{(1)}$.

La commission F\&P tient à remercier l'ensemble des sponsors qui ont permis l'organisation de ce minicolloque : Femmes \& Sciences, Triangle de la Physique, Dynamique des Systèmes Complexes. 